\title{
Patients' Perception towards General and Pelvic Examination by Male Medical Students: A Cross- sectional Study in a Multi-ethnic Population
}

\author{
Valliammai Jayanthi Thirunavuk Arasoo ${ }^{a}$, Nisha Angela Dominic ${ }^{a}$, Vanassa Ratnasingam ${ }^{a}$, Md Inzamum

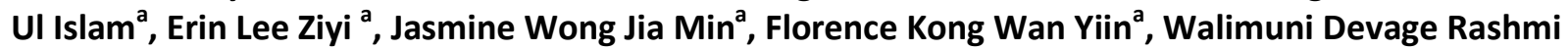 \\ Himashi Pemasiri ${ }^{a}$, Ravichandran Jeganathan ${ }^{b}$, Amutha Ramadas ${ }^{a}$ \\ ${ }^{a}$ Clinical School Johor Bahru, Monash University Malaysia, Johor Bahru, Malaysia \\ ${ }^{b}$ Hospital Sultanah Aminah Johor Bahru, Malaysia
}

\author{
Keywords \\ patients' perception; male medical students; \\ pelvic examination \\ Corresponding Author \\ Valliammai Javanthi Thirunavuk Arasoo, \\ Clinical School Johor Bahru, Monash \\ University Malaysia, 8 Jalan Masjid Abu \\ Bakar, 80100 Johor Bahru, Malaysia. \\ Tel:+607-2190600 \\ Tel No: +60391458782/ +60193750153 \\ Email: t.jayanthi@monash.edu \\ Received: 11 March 2020; Accepted: 20 \\ April 2021 \\ Doi: https://doi.org/10.31436/imjm.v20i4
}

\begin{abstract}
INTRODUCTION: : Male medical students globally have difficulty in obtaining consent to perform pelvic examinations. We sought to identify independent factors influencing women consenting to male medical students performing general and pelvic examinations under supervision. MATERIALS AND METHODS: This cross-sectional study was conducted at a tertiary hospital and a public health care centre in Johor Bahru, Malaysia where 369 women above 18 years old who have had sexual intercourse and no prior hysterectomy were recruited. A validated self-administered bilingual questionnaire was used to collect data on factors that influence them consenting to male medical students examining them and performing pelvic examination when indicated. RESULTS: The respondents were mostly below 30 years old (52.9\%), Malays (73.4\%), and Muslims $(75.3 \%)$. The consent rates for general and pelvic examination were $27.4 \%$ and $18.9 \%$, respectively. Being a Muslim, having an occupation, and being introduced by a male medical specialist increased women's likelihood of consenting to a general examination. However, a history of being examined by male students decreased the likelihood by $64 \%$. Believing that male doctors should have the skills to treat patients in women's health was the only independent factor that increased the likelihood for women to consent for pelvic examinations to be conducted by male medical students. CONCLUSION: Believing that male doctors should be skilled in treating women positively influences the decision to consent. Explaining earnestly to women how they aid in developing the skills of future doctors should be prioritised.
\end{abstract}

\section{INTRODUCTION}

Competency in performing pelvic examinations is essential in the clinical training of medical students. However, globally male medical students experience higher rates of declined consent to practice than their female counterparts. Male students felt their gender had a negative impact on their experience, but female students felt their gender had a positive impact. ${ }^{1,2}$

Overall, female students perform better when it comes to obtaining consent for pelvic examinations. History taking and examination by male students were acceptable, but intimate examination was less acceptable when attitudes to the gender of medical students by women in a gynaecology clinic were explored. ${ }^{3}$
The predominant Malay male students in a Malaysian medical school performed fewer pelvic examinations than their female colleagues. They also reported gender discrimination by medical officers and specialists. ${ }^{4}$ Despite male students reporting more embarrassment and a higher number of patient refusals in a United Kingdom school, they performed similar numbers of intimate examinations as the female students. The male students also felt clinical tutors were more likely to introduce patients to female students. ${ }^{5}$

In a Saudi Arabian study involving 492 patients, 80.7\% of patients felt that students' general appearance and manner affected their cooperation, and $57.9 \%$ preferred medical students of the same sex. $64 \%$ would not object to students' 
presence during an examination. In contrast, 38\% would accept the presence of both physician and medical students. Maintaining privacy was the most crucial aspect of a patientdoctor relationship. ${ }^{6}$

Patients seen by a female student and those who had prior student involvement reported more comfort in student participation. ${ }^{7}$ Some male students resort to honing their pelvic examinaation skills by examining patients under anaesthesia, but only 55\% of Canadian women in a study expected a medical student to be present during their surgery. A vast majority wanted to be informed of medical students' presence; $62 \%$ would consent to medical students performing pelvic examinations, and an additional 5\% allowing only female students. ${ }^{8}$ Patients under anaesthesia are indeed easier to examine as the pelvic musculature is relaxed. Although this is an established practice in Canada, physicians should be trained in the examination's conduct. ${ }^{?}$

Factors contributing to this refusal can be differentiated into modifiable viz professional approach, presence of chaperone and non-modifiable factors viz male gender, socio-cultural beliefs. We sought to identify patients' perception of modifiable factors to increase male medical students opportunities to hone their clinical skills.

\section{MATERIALS AND METHODS}

This cross-sectional study was conducted at Hospital Sultanah Aminah Johor Bahru (HSAJB) and Klinik Kesihatan Tampoi, Johor Bahru, Malaysia, from June 2018 to October 2019. The research protocol was approved by the Malaysian Research Ethics Committee (NMRR Research ID: 39896) and Monash University Research Ethics Committee (MUHREC ID: 14384) before study commencement.

A minimum sample size of 369 was calculated using OpenEPI, based on O'Flynn \& Rymer (2002) findings. Literate patients above 18 years of age who had had sexual intercourse before and had no prior hysterectomy were recruited from the Specialist O\&G Clinic, Antenatal Ward, Patient Assessment Unit of HSAJB, Gynaecology Ward, and Tampoi Community Health Clinic. Written consent from the participants were obtained prior to data collection.

With no suitable questionnaires catering to our local context, a questionnaire to assess modifiable and non-modifiable factors contributing to general and pelvic examination consent was developed. A draft questionnaire in the English language was developed using evidence gathered from a literature search and seeking expert opinion.
The study questionnaire consisted of the following sections: (I) demography (6 items); (II) clinical characteristics ( 9 items); (III) consent to examination ( 2 items, yes $=1$, no $=0$ ) and (IV) factors that influence consent to examination by male students ( 14 items, yes $=1$, no $=0$ and inverse scoring for negatively worded items).

Face validation of the draft was conducted among a group of experts in the area. Different individuals proficient in English and Malay languages performed a forward translation of the questionnaire to Malay, Malaysia's national language, and backward translation to English. The bilingual study questionnaire was piloted with 20 women for each language. A retest of the questionnaire was done in the same group of respondents one month later.

The internal consistency of Section (IV) was acceptable (Cronbach Alpha=0.732). We conducted McNemar test to compare the responses between test and retest questionnaires and found no statistical differences (all $\mathrm{p}>0.05$ ).

\section{Global comparison of patients' preference and consent rates}

A systematic literature search and review was conducted from June - July 2020 using Ovid Medline, EMBASE, and PubMed databases. Keyword combinations used in the search strategy included:[(gynaecological exam) OR (pelvic exam) OR (vaginal exam)] AND [(consent) OR (comfort) OR (preference)] AND (medical students). No language, geographical, or publication year limitations were imposed. Titles and abstracts of the studies obtained $(n=38)$ were then reviewed. At this stage, studies that did not report rate $(\%)$ of consent, preference, or comfort to general and/or pelvic examination specifically by female/male medical students were excluded. The reference lists of relevant articles were also manually searched to identify potential eligible articles that may have been missed during the electronic search. A total of 13 relevant studies were selected for the final inclusion. $3,7,11,12,25,30-37$.

\section{STATISTICAL ANALYSIS}

Statistical analysis was performed with IBM SPSS Statistics version 25.0. Data is described in frequencies and percentages. Association between patient demographic and clinical characteristics and characteristics of students, clinical scenario, past experience and social support, and consent rates were performed using Chi-square or Fisher's exact test. We conducted multivariate logistic regression to determine 


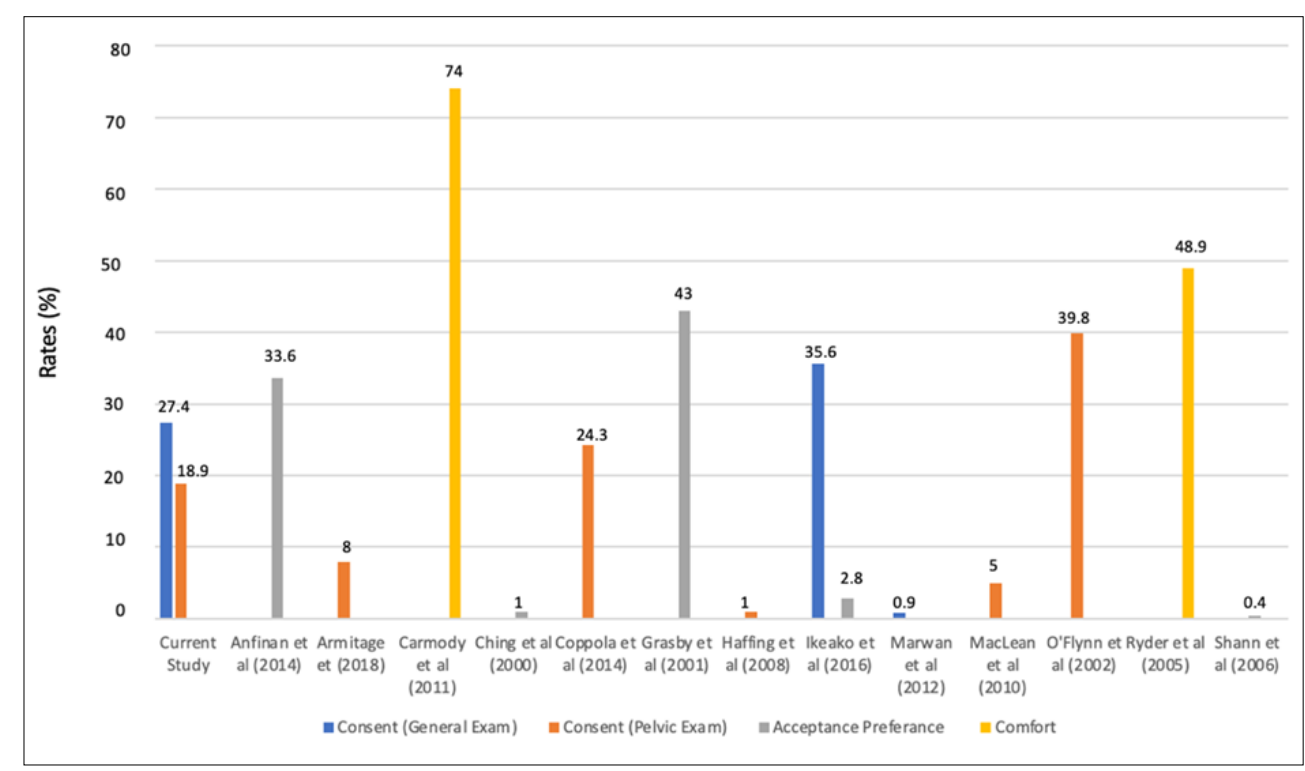

Figure 1: Global rates of consent, preference, acceptance, or comfort of female patients towards male medical students

the independent factors influencing women to allow general and pelvic examinations by male medical students. All statistical significance was set at $\mathrm{p}<0.05$.

\section{RESULTS}

The respondents were mostly below 30 years old $(52.9 \%)$, Malays (73.4\%), Muslims (75.3\%), have at least secondary level education $(96.7 \%)$, working $(58.4 \%)$, and married (92.6\%). Only $27.4 \%$ of the respondents were willing to consent to general examination by male medical students, and an even smaller proportion of them agreed to consent to pelvic examination $(18.9 \%)$. The global consent rates in the literature search for general examination by male medical students ranged from $0.9 \%$ to $35.6 \%$, while consent rates for pelvic examination ranged from $1 \%$ to $39.8 \%$. The acceptance/preference rates were also lower for male medical students, with the highest being $43 \%$ compared to $54 \%$ for female medical students. Comfort rates among participants were also lower for male medical students ( $74 \%$ vs. $86 \%$ ).

In our study, the univariate analysis (Table 1) showed a significant association between consent to general examination by male medical students and ethnicity $(p=0.011)$, religion $(p=0.011)$, and working status $(p=0.040)$. Interestingly none of these demographic indicators were associated with consent for a pelvic examination..

More than $40 \%$ of the respondents were recruited from obstetric wards, followed by primary care clinics (20.5\%) and gynaecology ward $(20.3 \%)$. More than $62 \%$ of the women were pregnant, with $75 \%$ in the third trimester. Almost $76 \%$ of them have children, and about one-third of the respondents have experienced at least one miscarriage in their lifetime. Most of the respondents (59.6\%) have experienced at least one vaginal delivery, while about $24 \%$ have experienced at least one caesarean delivery. Surprisingly, none of the clinical characteristics were associated with the consent to general and pelvic examinations by male medical students (Table 2).

Univariate analysis showed several factors that may influence women to allow general and pelvic examination by male medical students (Table 3). A student's characteristics such as being well-groomed $(p=0.022)$, politeness $(p=0.035)$, and use of similar language $(\mathrm{p}=0.035)$ were significantly associated with consent to general examination by male medical students. Consent to general examination was also related to several clinical scenarios ie student accompanied by a female chaperone $(\mathrm{p}=0.020)$, introduced by a male specialist $(\mathrm{p}<0.001)$, co-examined and treated by a senior doctor $(\mathrm{p}=0.008)$, and belief that male doctors required the skills in Women's Health $(\mathrm{p}=0.008)$.

Past experience of having examinations done by male doctors $(p=0.010)$ and male medical students $(p<0.001)$ also may be associated with consent to general examination. Conversely, the univariate analysis suggests that women felt coerced if asked by a senior doctor for consent to pelvic examination by male medical students $(\mathrm{p}=0.032)$. However, some women may also consent to pelvic examination as they believe male doctors require the skills $(\mathrm{p}=0.023)$. 
Table 1: Distribution of consent rates according to demographic characteristics of study respondents $(n=365)$

\begin{tabular}{|c|c|c|c|c|c|c|c|}
\hline & \multirow{2}{*}{$\begin{array}{l}\text { All respondents } \\
\qquad(\%) \\
(n=365)\end{array}$} & \multicolumn{2}{|c|}{$\begin{array}{c}\text { Consent to general exami- } \\
\text { nation by male students } \\
n(\%)\end{array}$} & \multirow[t]{2}{*}{$\mathbf{P}$} & \multicolumn{2}{|c|}{$\begin{array}{c}\text { Consent to pelvic examination by } \\
\text { male students } \\
\mathrm{n}(\%)\end{array}$} & \multirow[t]{2}{*}{$\mathbf{P}$} \\
\hline & & $\begin{array}{c}\text { Yes } \\
(n=100)\end{array}$ & $\begin{array}{c}\text { No } \\
(n=265)\end{array}$ & & $\begin{array}{c}\text { Yes } \\
(n=69)\end{array}$ & $\begin{array}{c}\text { No } \\
(n=296)\end{array}$ & \\
\hline \multicolumn{8}{|l|}{ Age (years) } \\
\hline$<30$ & 193 (52.9) & $54(54.0)$ & $139(52.5)$ & 0.790 & $38(55.1)$ & $155(52.4)$ & 0.623 \\
\hline $30-39$ & $140(38.4)$ & $36(36.0)$ & $104(39.2)$ & & $27(39.1)$ & $113(38.2)$ & \\
\hline$\geq 40$ & $32(8.8)$ & $10(10.0)$ & $22(8.3)$ & & $4(5.8)$ & $28(9.5)$ & \\
\hline \multicolumn{8}{|l|}{ Ethnicity } \\
\hline Malay & $268(73.4)$ & $64(64.0)$ & $204(77.0)$ & $0.011^{*}$ & 58 (84.1) & $210(70.9)$ & 0.097 \\
\hline Chinese & $28(7.7)$ & $10(10.0)$ & $18(6.8)$ & & $4(5.8)$ & $24(8.1)$ & \\
\hline Indian & $55(15.1)$ & $24(24.0)$ & $31(11.7)$ & & $7(10.1)$ & $48(16.2)$ & \\
\hline Others & $14(3.8)$ & $2(2.0)$ & $12(4.5)$ & & $0(0.0)$ & $14(4.7)$ & \\
\hline \multicolumn{8}{|l|}{ Religion } \\
\hline Muslim & $275(75.3)$ & $66(66.0)$ & $209(78.9)$ & $0.011^{*}$ & $58(84.1)$ & $217(73.3)$ & 0.062 \\
\hline Non-Muslim & $90(24.7)$ & $34(34.0)$ & $56(21.1)$ & & $11(15.9)$ & $79(26.7)$ & \\
\hline \multicolumn{8}{|l|}{ Education } \\
\hline $\begin{array}{l}\text { No formal } \\
\text { education }\end{array}$ & $2(0.5)$ & $0(0.0)$ & $2(0.8)$ & 0.974 & $0(0.0)$ & $2(0.7)$ & 0.806 \\
\hline Primary & $10(2.7)$ & $2(2.0)$ & $8(3.0)$ & & $2(2.9)$ & $8(2.7)$ & \\
\hline Secondary & $180(49.3)$ & $50(50.0)$ & $130(49.1)$ & & 37 (53.6) & $143(48.3)$ & \\
\hline Tertiary & $173(47.4)$ & $48(48.0)$ & 125 (47.2) & & $30(43.5)$ & 143 (48.3) & \\
\hline \multicolumn{8}{|l|}{ Occupation } \\
\hline No & 152 (41.6) & $33(33.0)$ & $119(44.9)$ & $0.040^{*}$ & $31(44.9)$ & $121(40.9)$ & 0.539 \\
\hline Yes & $213(58.4)$ & $67(67.0)$ & $146(55.1)$ & & 38 (55.1) & $175(59.1)$ & \\
\hline \multicolumn{8}{|l|}{ Marital status } \\
\hline Single & $23(6.3)$ & $6(6.0)$ & $17(6.4)$ & 0.573 & $5(7.2)$ & $18(6.1)$ & 0.818 \\
\hline Married & 338 (92.6) & $92(92.0)$ & $246(92.8)$ & & $64(92.8)$ & $274(92.6)$ & \\
\hline $\begin{array}{l}\text { Widowed/ } \\
\text { divorced }\end{array}$ & $4(1.1)$ & $2(2.0)$ & $2(0.8)$ & & $0(0.0)$ & $4(1.4)$ & \\
\hline
\end{tabular}

Data presented as $\mathrm{n}(\%)$ and analysed with chi square or fisher's exact test *significant at $\mathrm{p}<0.05$

We conducted a further analysis with multivariate logistic regression to identify independent predictors of consent to general examination and pelvic examination (Table 4) by male medical students. Consent to general examination was examined for its association with demography (ethnicity, religion, and occupation), student's characteristics (wellgroomed, politeness, confidence, similar language), clinical setting (accompanied by a female chaperone, introduced by a male medical specialist, co-examined and treated by a senior doctor), past experience and husband/partner's influence on their decision. Being of a Muslim background increases the likelihood of giving consent to general examination by male medical students $(\mathrm{AOR}=2.14,95 \% \mathrm{CI}=1.23-3.71, \mathrm{p}=0.007)$ while working women are $72 \%$ more likely to provide Health. consent $\quad(\mathrm{AOR}=1.72, \quad 95 \% \quad \mathrm{CI}=1.03-2.87, \quad \mathrm{p}=0.039)$. Introduction by a male specialist increased the odds of consent by two times $(\mathrm{AOR}=2.06,95 \% \quad \mathrm{CI}=1.14-3.73$, $\mathrm{p}=0.016$ ). However, history or examination by male students decreased the likelihood for women to provide consent to general examination by $64 \%(\mathrm{AOR}=0.36,95 \% \mathrm{CI}=0.22-0.59$, $\mathrm{p}<0.001)$.

Consent to pelvic examination by male medical students was explored regarding its association with ethnicity, religion, feeling coerced to consent if asked by a senior doctor, coexamined and treated by a senior doctor, and belief that male doctors should have the skills to treat patients in Women's 
Table 2: Distribution of consent rates according to clinical characteristics of study respondents $(n=365)$

\begin{tabular}{|c|c|c|c|c|c|c|c|}
\hline & \multirow{2}{*}{$\begin{array}{l}\text { All respondents } \\
\text { n(\%) } \\
\\
(n=365)\end{array}$} & \multicolumn{2}{|c|}{$\begin{array}{c}\text { Consent to general examination by } \\
\text { male students } \\
\mathrm{n}(\%)\end{array}$} & \multirow[t]{2}{*}{$\mathbf{P}$} & \multicolumn{2}{|c|}{$\begin{array}{c}\text { Consent to pelvic examination } \\
\text { by male students } \\
\mathrm{n}(\%)\end{array}$} & \multirow[t]{2}{*}{$\mathbf{P}$} \\
\hline & & $\begin{array}{c}\text { Yes } \\
(n=100)\end{array}$ & $\begin{array}{c}\text { No } \\
(n=265)\end{array}$ & & $\begin{array}{c}\text { Yes } \\
(\mathrm{n}=69)\end{array}$ & $\begin{array}{c}\text { No } \\
(n=296)\end{array}$ & \\
\hline \multicolumn{8}{|l|}{ Source } \\
\hline $\begin{array}{l}\text { Primary } \\
\text { care clinic }\end{array}$ & $75(20.5)$ & $24(24.0)$ & $51(19.2)$ & 0.271 & $18(26.1)$ & $57(19.3)$ & 0.551 \\
\hline SCOG & $67(18.4)$ & $15(15.0)$ & $52(19.6)$ & & $12(17.4)$ & $55(18.6)$ & \\
\hline Gynae ward & $74(20.3)$ & $26(26.0)$ & $48(18.1)$ & & $11(15.9)$ & $63(21.3)$ & \\
\hline $\begin{array}{l}\text { Obstetric \& } \\
\text { antenatal }\end{array}$ & $149(40.8)$ & $35(35.0)$ & $114(43.0)$ & & $28(40.6)$ & $121(40.9)$ & \\
\hline \multicolumn{8}{|c|}{ Pregnancy experience } \\
\hline Never pregnant & $26(7.1)$ & $9(9.0)$ & $17(6.4)$ & 0.678 & $3(4.3)$ & $23(7.8)$ & 0.544 \\
\hline $\begin{array}{l}\text { Currently } \\
\text { pregnant }\end{array}$ & $228(62.5)$ & $62(62.0)$ & $166(62.6)$ & & $46(66.7)$ & $182(61.5)$ & \\
\hline $\begin{array}{l}\text { Previously } \\
\text { pregnant }\end{array}$ & $111(30.4)$ & $29(29.0)$ & $82(30.9)$ & & $20(29.0)$ & $91(30.7)$ & \\
\hline \multicolumn{8}{|l|}{ Trimester } \\
\hline First & $22(11.2)$ & $6(11.1)$ & $16(11.3)$ & 0.489 & $6(15.0)$ & $16(10.3)$ & 0.347 \\
\hline Second & $27(13.8)$ & $10(18.5)$ & $17(12.0)$ & & $3(7.5)$ & $24(15.4)$ & \\
\hline Third & $147(75.0)$ & $38(70.4)$ & $109(76.8)$ & & $31(77.5)$ & $116(74.4)$ & \\
\hline \multicolumn{8}{|l|}{ Children $^{\text {b }}$} \\
\hline None & $83(24.5)$ & $24(26.4)$ & $59(23.8)$ & 0.669 & $16(24.2)$ & $67(24.5)$ & 0.959 \\
\hline Yes & $256(75.5)$ & $67(73.6)$ & $189(76.2)$ & & $50(75.8)$ & $206(75.5)$ & \\
\hline \multicolumn{8}{|l|}{ Miscarriages ${ }^{\mathrm{b}}$} \\
\hline None & $234(69.0)$ & $62(68.1)$ & $172(69.4)$ & 0.829 & $47(71.2)$ & $187(68.5)$ & 0.669 \\
\hline Yes & $105(31.0)$ & $29(31.9)$ & $76(30.6)$ & & $19(28.8)$ & $86(31.5)$ & \\
\hline \multicolumn{8}{|c|}{ Vaginal delivery ${ }^{\mathrm{b}}$} \\
\hline None & $137(40.4)$ & $39(42.9)$ & $98(39.5)$ & 0.579 & $25(37.9)$ & $112(41.0)$ & 0.640 \\
\hline Yes & $202(59.6)$ & $52(57.1)$ & $150(60.5)$ & & $41(62.1)$ & $161(59.0)$ & \\
\hline \multicolumn{8}{|c|}{ Caesarean delivery ${ }^{\mathrm{b}}$} \\
\hline None & $259(76.4)$ & $72(79.1)$ & $187(75.4)$ & 0.475 & $51(77.3)$ & $208(76.2)$ & 0.853 \\
\hline Yes & $80(23.6)$ & $19(20.9)$ & $61(24.6)$ & & $15(22.7)$ & $65(23.8)$ & \\
\hline
\end{tabular}

Data presented as $\mathrm{n}(\%)$ and analysed with chi square or fisher's exact test ${ }^{a}=196$; ${ }^{b}$ currently pregnant or ever pregnant

Of all these potential factors, only the belief that male doctors should have the skills was significantly associated with an increased likelihood of women to provide consent to pelvic examination $(\mathrm{AOR}=2.37,95 \% \mathrm{CI}=1.03-5.45, \mathrm{p}=0.043)$.

\section{DISCUSSION AND CONCLUSION}

This paper evaluates background characteristics and influencing factors that may lead to women consenting to male medical students performing general and pelvic examination when indicated. Overall, the percentage of women who will allow male students to perform these examinations is dismal, $27.4 \%$ and $18.9 \%$.
When asked for consent to perform a general examination, Muslim women were more likely to consent than women of different faiths $(\mathrm{p}=0.007)$. However, there was no difference when it came to consent for a pelvic examination. Regional papers looking at this aspect are limited. A study at Medical University South Carolina explored background characteristics found that Whites were more likely than Blacks to allow student participation. Hispanics and Asians were the least likely to do so. ${ }^{10}$

Women who work were likely to consent to general examination $(p=0.039)$ but not a pelvic examination. In Kuwait, a study showed that of the small number who agreed 
Table 3: Distribution of consent rates according to characteristics of students, clinical scenario, past experience and social support ( $\mathrm{n}=365$ )

\begin{tabular}{|c|c|c|c|c|c|c|c|c|}
\hline & & \multirow{2}{*}{$\begin{array}{c}\text { All } \\
\text { respondents } \\
n(\%) \\
(n=365)\end{array}$} & \multicolumn{2}{|c|}{$\begin{array}{c}\text { Consent to general examination } \\
\text { by male students } \\
n(\%)\end{array}$} & \multirow[t]{2}{*}{$\mathbf{P}$} & \multicolumn{2}{|c|}{$\begin{array}{l}\text { Consent to pelvic examination by } \\
\text { male students } \\
n(\%)\end{array}$} & \multirow[t]{2}{*}{$\mathbf{P}$} \\
\hline & & & $\begin{array}{c}\text { Yes } \\
(n=100)\end{array}$ & $\begin{array}{c}\text { No } \\
(n=265)\end{array}$ & & $\begin{array}{c}\text { Yes } \\
(n=69)\end{array}$ & $\begin{array}{c}\text { No } \\
(n=296)\end{array}$ & \\
\hline \multicolumn{9}{|c|}{ A) STUDENT'S CHARACTERISTICS } \\
\hline \multirow[t]{2}{*}{ Well-groomed } & Yes & $306(83.8)$ & $91(91.0)$ & $215(81.1)$ & $0.022^{*}$ & $60(87.0)$ & $246(83.1)$ & 0.434 \\
\hline & No & $59(16.2)$ & $9(9.0)$ & $50(18.9)$ & & $9(13.0)$ & $50(16.9)$ & \\
\hline \multirow[t]{2}{*}{ Politeness } & Yes & $322(88.2)$ & $94(94.0)$ & $228(86.0)$ & $0.035^{*}$ & $62(89.9)$ & $260(87.8)$ & 0.640 \\
\hline & No & $43(11.8)$ & $6(6.0)$ & $37(14.0)$ & & $7(10.1)$ & $36(12.2)$ & \\
\hline \multirow[t]{2}{*}{ Confidence } & Yes & $326(89.3)$ & $94(94.0)$ & $232(87.5)$ & 0.075 & $64(92.8)$ & $262(88.5)$ & 0.305 \\
\hline & No & $39(10.7)$ & $6(6.0)$ & $33(12.5)$ & & $5(7.2)$ & $34(11.5)$ & \\
\hline \multirow[t]{2}{*}{ Language } & Yes & $322(88.2)$ & $94(94.0)$ & $228(86.0)$ & $0.035^{*}$ & $63(91.3)$ & $259(87.5)$ & 0.377 \\
\hline & No & $43(11.8)$ & $6(6.0)$ & $37(14.0)$ & & $6(8.7)$ & $37(12.5)$ & \\
\hline \multirow[t]{2}{*}{ Same ethnicity } & Yes & $169(46.3)$ & $46(46.0)$ & $123(46.4)$ & 0.943 & $31(44.9)$ & 138 (46.6) & 0.799 \\
\hline & No & $196(53.7)$ & $54(54.0)$ & $142(53.6)$ & & 38 (55.1) & $158(53.4)$ & \\
\hline \multirow[t]{2}{*}{ Same religion } & Yes & $162(44.4)$ & $43(43.0)$ & $119(44.9)$ & 0.744 & $31(44.9)$ & $131(44.3)$ & 0.920 \\
\hline & No & $203(55.6)$ & $57(57.0)$ & $146(55.1)$ & & $38(55.1)$ & $165(55.7)$ & \\
\hline \multicolumn{9}{|c|}{ B) CLINICAL SCENARIO } \\
\hline \multirow{2}{*}{$\begin{array}{l}\text { Accompanied by a } \\
\text { female chaperone }\end{array}$} & Yes & $310(84.9)$ & $92(92.0)$ & $218(82.3)$ & $0.020^{*}$ & $60(87.0)$ & $250(84.5)$ & 0.602 \\
\hline & No & $55(15.1)$ & $8(8.0)$ & 47 (17.7) & & $9(13.0)$ & $46(15.5)$ & \\
\hline \multirow{2}{*}{$\begin{array}{l}\text { Introduced by } \\
\text { female nurse/ } \\
\text { medical personnel }\end{array}$} & Yes & $313(85.8)$ & $87(87.0)$ & $226(85.3)$ & 0.676 & $61(88.4)$ & $252(86.1)$ & 0.484 \\
\hline & No & $52(14.2)$ & $13(13.0)$ & $39(14.7)$ & & $8(11.6)$ & $44(14.9)$ & \\
\hline \multirow{2}{*}{$\begin{array}{l}\text { Introduced by a } \\
\text { male specialist }\end{array}$} & Yes & $236(64.7)$ & $79(79.0)$ & $157(59.2)$ & $<0.001^{* *}$ & 48 (69.6) & $188(63.5)$ & 0.344 \\
\hline & No & $129(35.3)$ & $21(21.0)$ & $108(40.8)$ & & $21(30.4)$ & $108(36.5)$ & \\
\hline \multirow{2}{*}{$\begin{array}{l}\text { Introduced by a } \\
\text { female specialist }\end{array}$} & Yes & $310(84.9)$ & $86(86.0)$ & $224(84.5)$ & 0.726 & $60(87.0)$ & $250(84.5)$ & 0.602 \\
\hline & No & 55 (15.1) & $14(14.0)$ & $41(15.5)$ & & $9(13.0)$ & $46(15.5)$ & \\
\hline \multirow{2}{*}{$\begin{array}{l}\text { Felt coerced as } \\
\text { asked by a senior } \\
\text { doctor }\end{array}$} & Yes & $197(54.0)$ & $49(49.0)$ & $148(55.8)$ & 0.242 & $30(43.5)$ & $167(56.4)$ & $0.032 *$ \\
\hline & No & $168(46.0)$ & $51(51.0)$ & $117(44.2)$ & & $39(56.5)$ & $129(43.6)$ & \\
\hline \multirow{4}{*}{$\begin{array}{l}\text { Co-examined and } \\
\text { treated by a senior } \\
\text { doctor } \\
\text { Male doctors } \\
\text { require the skills }\end{array}$} & Yes & $238(65.2)$ & $76(76.0)$ & $162(61.1)$ & $0.008^{*}$ & $51(73.9)$ & $187(63.2)$ & 0.092 \\
\hline & No & $127(34.8)$ & $24(24.0)$ & $103(38.9)$ & & $18(26.1)$ & $109(36.8)$ & \\
\hline & Yes & $292(80.0)$ & $89(89.0)$ & $203(76.6)$ & $0.008^{*}$ & $62(89.9)$ & $230(77.7)$ & $0.023^{*}$ \\
\hline & No & $73(20.0)$ & $11(11.0)$ & $62(23.4)$ & & $7(10.1)$ & $66(22.3)$ & \\
\hline \multicolumn{9}{|c|}{ C) PAST EXPERIENCE } \\
\hline \multirow{2}{*}{$\begin{array}{l}\text { History of } \\
\text { examination by } \\
\text { male doctors }\end{array}$} & Yes & $280(76.7)$ & $86(86.0)$ & $194(73.2)$ & $0.010^{*}$ & $53(76.8)$ & $227(76.7)$ & 0.983 \\
\hline & No & $85(23.3)$ & $14(14.0)$ & $71(26.8)$ & & $16(23.2)$ & $69(23.3)$ & \\
\hline \multirow{2}{*}{$\begin{array}{l}\text { History of } \\
\text { examination by } \\
\text { male medical } \\
\text { students }\end{array}$} & Yes & $125(34.2)$ & $52(52.0)$ & $73(27.5)$ & $<0.001 * *$ & $20(29.0)$ & $105(35.5)$ & 0.306 \\
\hline & No & $240(65.8)$ & $48(48.0)$ & $192(72.5)$ & & $49(71.0)$ & $191(64.5)$ & \\
\hline \multicolumn{9}{|c|}{ D) SOCIAL SUPPORT } \\
\hline \multirow{2}{*}{$\begin{array}{l}\text { Husband's / } \\
\text { partner's opinion }\end{array}$} & Yes & $205(56.2)$ & $63(63.0)$ & $142(53.6)$ & 0.106 & $37(53.6)$ & $168(56.8)$ & 0.637 \\
\hline & No & $160(43.8)$ & $37(37.0)$ & $123(46.4)$ & & $32(46.4)$ & $128(43.2)$ & \\
\hline
\end{tabular}

Data presented as $\mathrm{n}(\%)$ and analysed with chi square test

*significant at $\mathrm{p}<0.05 ; * *$ significant at $\mathrm{p}<0.001$ 
to students examining them, occupation status was significant. ${ }^{11}$

Past experience of having examinations done by male doctors $(p=0.010)$ and male medical students $(p<0.001)$ also may be associated with consent to general examination but not pelvic examination. Armitage and Cahil's analysis of questionnaires completed by 233 women on consenting to pelvic examination by medical students of either gender indicated that although age, parity and marital status had no bearing on a woman's willingness to consent to pelvic examination, attending a gynaecology clinic before positively affected willingness to consent. ${ }^{12}$

Table 4: Independent predictors of female respondents' consent to general and pelvic examinations by male medical students $(n=365)$

\begin{tabular}{lccc}
\hline & $\begin{array}{c}\text { Adjusted } \\
\text { OR }\end{array}$ & 95\% CI & P \\
\hline General examination & & & \\
\hline Demography & & & \\
Muslim & 2.14 & $1.23-3.71$ & $0.007^{*}$ \\
Working & 1.72 & $1.03-2.87$ & $0.039^{*}$ \\
\hline Clinical scenario & & & \\
$\begin{array}{l}\text { Accompanied by } \\
\text { female chaperone }\end{array}$ & 2.24 & $0.93-5.38$ & 0.071 \\
$\begin{array}{l}\text { Introduced by male } \\
\text { specialist }\end{array}$ & 2.06 & $1.14-3.73$ & $0.016^{*}$ \\
\hline
\end{tabular}

\begin{tabular}{llll}
\hline Past Experience & & & \\
$\begin{array}{l}\text { History or examination } \\
\text { by male medical } \\
\text { student }\end{array}$ & 0.36 & $0.22-0.59$ & $<0.001^{* *}$ \\
\hline
\end{tabular}

\begin{tabular}{|c|c|c|c|}
\hline \multicolumn{4}{|l|}{ Pelvic examination } \\
\hline \multicolumn{4}{|l|}{ Clinical scenario } \\
\hline $\begin{array}{l}\text { Felt coerced when } \\
\text { asked by a senior } \\
\text { doctor }\end{array}$ & 0.80 & $0.61-1.04$ & 0.098 \\
\hline $\begin{array}{l}\text { Male doctors } \\
\text { require the skills }\end{array}$ & 2.37 & $1.03-5.45$ & $0.043^{*}$ \\
\hline
\end{tabular}

The consensus from a general public survey in Western Australia was general examination by medical students was largely acceptable but permission for intimate examinations if at all given was restricted to advanced medical students. Those who gave consent were more likely to have been hospitalised or been examined by medical students before. ${ }^{13}$

The majority of our participants were married and being married was not a characteristic of them consenting to general or pelvic examination by male students. Our finding that parity and a prior vaginal delivery were not influencing factors was aligned with the results of a study ${ }^{12}$ but contrasted with other studies that noted parous women were more likely to allow student participation than nulliparous women. 3,10
There were also no differences in inpatient versus outpatient settings or specialist outpatient clinics versus public health clinics. No particular setting had participants who were more likely to consent to both general and pelvic examination. In 2017, participants from similar backgrounds as ours were surveyed in a hospital in Saudi Arabia. However, their gynaecological patients were more accepting of medical students than obstetric patients. ${ }^{14}$ Studies conducted elsewhere reported that male students are denied consent to perform pelvic examination more often by clinic patients than by private patients. ${ }^{15,16}$ None of our participants are private patients.

As for the general appearance, politeness, and how confident a male student is, our participants' response showed that although being well-groomed $(p=0.22)$ and polite $(p=0.035)$ were positive influencing factors for general examination, they were not for a pelvic examination. The appearance of medical students and whether they spent time building rapport with respondents can be positive influencers ${ }^{12}$ and did influence patients' willingness to allow students to participate in their care in Riyadh. ${ }^{6}$

Across all ethnic groups in an urban multi-ethnic community with a racially diverse population of obstetricians, a strong racial bias in the patient selection of an obstetrician was noted. ${ }^{17}$ Our participants, however, said ethnic or religious concordance did not make a difference. Although communicating in a language the participant is comfortable with was significant $(p=0.035)$ for respondents' consenting to general examination, it was not an independent predictor. The language used did not influence obtaining consent for a pelvic examination. Another study noted that clinical competence or communication skills were more important than the gender of the care provider. ${ }^{18}$

Seeking consent when female medical personnel chaperone the student or when a male specialist does the introduction were significant independent predictors for respondents consenting to general examination by male students but not for pelvic examination in our study. Tang and Skye studied the influence of preceptors. Their respondents were more likely to decline student involvement when preceptors used another medical team member such as medical assistants, physician assistants, or nurses to obtain permission to have a medical student involved in their care. ${ }^{19}$ In education and training settings, a female member of the team does improve the possibility of male residents participating in gynaecologic examination as women are uncomfortable with the presence of males in gynaecological examination and have difficulty discussing sexual matters with a man. More importantly, compassion and communication help alleviate embarrassment. ${ }^{20}$ These show the importance of 
acknowledging a patient's potential discomfort before performing a pelvic examination and teaching gendersensitive communication style.

Our participants reported feeling coerced into consenting for pelvic examination if approached by a senior doctor, but when independent predictors were analysed, this was not statistically significant $(p=0.098)$. They were also likely to consent if the male student performs the pelvic examination under a senior doctor's supervision. Students may attempt to request consent by linking it to care provided by supervising clinicians, which reduces patient freedom to choose. ${ }^{21}$ When determining the element of coercion in obtaining permission, $86 \%$ of 226 participants studied by Berry et al. said they would prefer a nurse instead of a physician or student approaching them for consent. This approach would reduce coercion taking place. ${ }^{22}$

Malaysia is a multi-ethnic and conservative country. ${ }^{23}$ Interestingly, despite being part of a conservative society, the husband's opinion did not influence the participant's decision to consent to both general and pelvic examination. The majority of the participants are Muslims (74.5\%) with secondary school education $(49.6 \%)$ or tertiary education $(47.2 \%)$. Education may be the reason for their independent decision-making as a study in Ghana showed that of the 94 Muslim women interviewed, those with lower formal education obeyed their husbands' decisions when it came to birthing in hospitals. ${ }^{24}$

Fortunately, women of all religious faiths believe that male doctors should have skills to treat patients in Women's Health $(p=0.043)$. Emirati women too acknowledged responsibility in the training of all their medical students. ${ }^{25}$ In a commentary by Ameer $\mathrm{Z}$ Aldee, the following issues were highlighted. ${ }^{26}$ When treatment is required for non-emergent conditions, most Islamic scholars believe that the order of preference for providers should be Muslims of the same gender, followed by non-Muslims of the same gender, Muslims of the opposite gender, and finally Muslims of the opposite gender. However, there is no religious opposition to complete examination by a physician of the opposite gender in emergent care. ${ }^{27} \mathrm{~A}$ proper explanation to women that they are helping doctors of the future acquire skills needed in emergencies will be helpful.

Medical schools in some developed countries that use women trained to teach pelvic examination while being examined have shown that some students' anxieties may be relieved. ${ }^{28}$ This teaching strategy involves cost and may not be an economically viable option for most of the 202 medical schools in Southeast Asia ${ }^{29}$ but maybe a way forward to improve male medical students' clinical skills and confidence.
This study's strengths include the large sample size recruited from different clinical locations, including both obstetrics and gynaecology patients. The limited number of similar studies from the South East Asian region hindered making reasonable comparisons.

The patients' perspective is pivotal as it directs the area to focus on to improve the learning experience of male students in performing pelvic examinations. The belief that male doctors should have the skills to examine a woman entirely means effort should be spent in explaining to women the importance of having male doctors who are competent in performing a pelvic examination and how they can help achieve this. Further studies should be conducted to explore suggestions by women themselves on ways to improve male students' physical examination learning process.

\section{Limitations of the study}

The authors acknowledge concluding that Malay or Muslim women are more likely to give consent compared to other race and religions are not suitably represented by this research. There was no oversampling of non-Malay or nonMuslims, but the study design conformed to the usual demography of women who seek treatment in a public Malaysian healthcare setting. The team decided to screen and recruit women who consented to the study when approached. To ensure an even distribution across race and religion an oversampling the non-Malay or non-Muslim will reduce this bias.

\section{ACKNOWLEDGEMENTS}

The team of authors would like to acknowledge the staff members of the Department of Obstetrics \& Gynaecology, Hospital Sultanah Aminah Johor Bahru and the staff members of Klinik Kesihatan Tampoi, Johor Bahru for their kind assistance in this project

\section{DECLARATIONS}

Ethics approval : The research protocol was approved by the Malaysian Research Ethics Committee (upon reasonable request to the corresponding author NMRR Research ID: 39896) and Monash University Research Ethics Committee (MUHREC ID: 14384) prior to study commencement.

\section{REFERENCES}

1. Emmons SL, Adams KE, Nichols M, Cain J. 'The impact of perceived gender bias on obstetrics and gynaecology skills acquisition by third-year medical students'. 
Academic Medicine. 2004 Apr; 79(4): 326-32

2. Bhoopatkar H, Wearn A and Vnuk A. 'Medical Student's experience of performing female pelvic examinations: Opportunities and Barriers'. The Australian and New Zealand Journal of Obstetrics and Gynaecology 2017: 57: 514-519

3. O'Flynn N, Rymer J. 'Women's attitudes to the sex of medical students in a gynaecology clinic: cross sectional survey'. BMJ: The British Medical Journal, 2002; 325: 683-4

4. Zahid AZ, Ismail Z, Abdullah B, Daud S. 'Gender bias in training of medical students in obstetrics and gynaecology: A myth or reality?'. European Journal of Obstetrics \& Gynecology and Reproductive Biology 2015; 186:17-21

5. Akkad A, Bonas S, Stark P. 'Gender differences in final year medical students' experience of teaching of intimate examinations: a questionnaire study'. BJOG: An International Journal of Obstetrics \& Gynaecology 2008;115(5):625-32

6. Abdulghani HM, Al-Rukban MO, Ahmad SS. 'Patient attitudes towards medical students in Riyadh, Saudi Arabia'. Education for Health Abingdon). 2008;21 (2):69.

7. Carmody D, Tregonning A, Nathan E, Newnham JP. 'Patient perceptions of medical students' involvement in their obstetrics and gynaecology health care'. Australian and New Zealand Journal of Obstetrics and Gynaecology. 2011; 51: 553-558

8. Wainberg S, Wrigley H, Fair J, Ross S. 'Teaching pelvic examinations under anaesthesia: what do women think?'. Journal of Obstetrics and Gynaecology Canada. 2010;32 (1):49-53.

9. Gibson E, Downie J. 'Consent requirements for pelvic examinations performed for training purposes'. Canadian Medical Association Journal. 012;184(10):1159 $-1161$.

10. Thurman AR, Litts PL, O'Rourke K, Swift S. 'Patient acceptance of medical student participation in an outpatient obstetric/gynecologic clinic'. The Journal of reproductive medicine, 2006, Vol.51(2), pp.109-14

11. Marwan Y, Al-Saddique M, Hassan A, Karim J, Al-Saleh M. 'Are medical students accepted by patients in teaching hospitals?'. Medical Education Online. 2012; 17:17172.

12. Armitage AJ, Cahill DJ. 'Medical students and intimate examinations: What affects whether a woman will consent?'. Medical Teacher, 2018: 40:12, 1281-1286

13. Koehler N, McMenamin C. Would you consent to being examined by a medical student? Western Australian general public survey’. Medical Teacher: 2012. 34:7, e518-e528

14. Subki AH, Algethami MR, Addas FA, Alnefaie MN,
Hindi MM, et al. 'Women's perception and attitude to medical students' participation in obstetrics and gynecology care'. Saudi Medical Journal, 2018: 39(9), 902-909.

15. Johnson AM, Schnatz. "Gender Preferences in Ob-Gyn Clinic vs Private clinic'. Connecticut Medicine 2009; 73 (4):205-209

16. Xuezhi J, Altomare, Christopher, Egan, James FX, et al. 'The ObGyn Clerkship: Are Students Denied the Opportunity to Provide Patient Care and What is the Role of Gender?'. Connecticut Medicine. 2012, 76(4), p231-236.

17. Schneider, Karen M, Strecker, Michelle N, Promecene, et al. 'Racial bias in patient selection of an obstetrician'. Southern Medical Journal, 2005. 98(4), 409-410.

18. Mavis B, Vasilenko P, Schnuth R, Marshall J, Jeff M. 'Female patients' preferences related to interpersonal communications, clinical competence, and gender when selecting a physician'. Academic Medicine: Journal of the Association of American Medical Colleges, 2005. 80 (12), 1159-1165.

19. Tang TS, Skye EP. 'Who Gets "Kicked Out" of the Exam Room? Factors Associated with Patients Declining Medical Student Participation'. Teaching and Learning in Medicine, 2009: 21:1, 1-7,

20. Rifkin JI, Shapiro HG, Regensteiner JK, Stotler J, Schmidt B. (2002). Why Do Some Women Refuse to Allow Male Residents to Perform Pelvic Exams?'. Academic Medicine (2002) 77(10), 1034-1038.

21. Carson-Stevens A, Davies MM, Jones R, Chik AD, Robbé IJ, et al. 'Framing patient consent for student involvement in pelvic examination: A dual model of autonomy'. Journal of Medical Ethics (2013) 39(11), 676.

22. Berry RE Jr, O'dell K, Meyer BA, Purwono U. 'Obtaining patient permission for student participation in obstetric-gynecologic outpatient visits: a randomized controlled trial'. American Journal of Obstetrics and Gynecology. 2003; 189(3):634-638.

23. Osman MNM. 'The Islamic conservative turn in Malaysia: impact and future trajectories'. Contemporary Islam (2017)11, 1-20.

24. Ganle JK. 'Why Muslim women in Northern Ghana do not use skilled maternal healthcare services at health facilities: a qualitative study'. BMC International Health and Human Rights. 2015; 15:10.

25. McLean $\mathrm{M}, \mathrm{Al}$ Ahbabi S, Al Ameri M, Al Mansoori M, Al Yahyaei, F. et al. Muslim women and medical students in the clinic encounter. Medical Education, (2010) 44: 306-315.

26. Aldeen AZ. 'Commentary: The Muslim ethical tradition and emergent medical care: an uneasy fit'. Academic Emergency Medicine. 2007;14(3):277-278. 
27. Al-Munajjid S. Islam. 'Questions and Answers, Question 5693’. Available at: http://www.islam-qa.com/index .

28. Grankvist O, Olofsson AD, Isaksson RM. 'Can physicians be replaced with gynecological teaching women to train medical students in their first pelvic examination? A pilot study from Northern Sweden'. Patient Education and Counseling, (2014) 96(1), 50 - 54

29. World Directory of Medical Schools. https:// search.wdoms.org/

30. Anfinan N, Alghunaim N, Boker A, Hussain, A, Almarstani A, et al. 'Obstetric and gynecologic patients' attitudes and perceptions toward medical students in Saudi Arabia', Oman Medical Journal, (2014) 29(2) pp.106-109.

31. Ching SL, Gates EA, Robertson PA. 'Factors influencing obstetric and gynecologic patients' decisions towards medical student involvement in the outpatient setting', American Journal of Obstetrics and Gynecology, (2000) 182(6) pp. 1429-1432.

32. Coppola LM, Reed KL, Herbert WN. 'Comparison of patient attitudes and provider perceptions regarding medical student involvement in obstetric/gynecologic care'. Teaching and Learning in Medicine, (2014) 26 (3) pp. 239-243.

33. Grasby D, Quinlivan JA. 'Attitudes of patients towards the involvement of medical students in their intrapartum obstetric care'. Australian and New Zealand Journal of Obstetrics and Gynaecology, (2001) 41(1) pp. 91-96.

34. Haffling A, Håkansson A. 'Patients consulting with students in general practice:Survey of patients' satisfaction and their role in teaching'. Medical Teacher, 2008: 30(6) pp. 622-629.

35. Ikeako LC, Adiuku-Brown A, Ezegwui HU, Onuh, AC, Okeke TC. 'Attitudes of patients to medical students in the gynaecology clinic: A Nigerian experience'. British Journal of Medicine \& Medical Research: (2016) 15(11) pp. 1-10.

36. Ryder N, Ivens D, Sabin C. 'The attitude of patients towards medical students in a sexual health clinic'. Sexually Transmitted Infections, (2005)81(5) pp. $437-$ 439.

37. Shann S, Wilson JD. 'Patients' attitudes to the presence of medical students in a genitourinary medicine clinic: a cross sectional survey'. Sexually Transmitted Infections, (2006) 82(1) pp. 52-54. 